\title{
Deformation Analysis of Forsterite Olivine Using Electron Channeling Contrast Imaging and Electron Backscatter Diffraction
}

\author{
Shirin Kaboli ${ }^{1}$, Raynald Gauvin ${ }^{2}$ and Pamela Burnley ${ }^{3}$ \\ 1,3. Department of Geoscience and High Pressure Science and Engineering Center, University of Nevada, \\ Las Vegas, Nevada (UNLV), Las Vegas, United States \\ 2. Department of Mining and Materials Engineering, McGill University, Montreal, Canada
}

The dynamics of the Earth system is expressed in a number of ways such as earthquakes, mountain building and plate tectonics. The rheology of Earth materials at the conditions of Earth's interior dictates the nature of these phenomena and therefore, is an important field of research in Earth sciences. The flow strength in rocks is a function of minerology, crystal defects substructures, grain size, shape and crystallographic orientations. Furthermore, flow strength in rocks strongly depends on temperature and pressure levels. Experimental deformation studies focus on understanding these various dependencies in rocks during deformation processes in Earth's interior. In particular, the in-situ synchrotron x-ray diffraction technique integrates the multi-anvil high-pressure system with the synchrotron in order to quantify plastic properties of Earth's materials at pressures relevant to the Earth's upper and lower mantle [1].

A detailed analysis of the microstructure and crystallographic texture of deformed rocks at micro- and nano-scale provide valuable information on plastic behavior of rocks during deformation. The electron channeling contrast imaging (ECCI) and electron backscatter diffraction (EBSD) techniques provide microstructural and quantitative crystallographic information on a bulk specimen in a scanning electron microscope (SEM). Under certain electron optics operating conditions in a field emission gun SEM, the secondary electron (SE) and backscattered electron (BSE) contrast include a contribution from the changes in crystallographic orientations of the specimen. The crystallographic contrast is superimposed on the normal topographical and compositional contrast. The contribution of crystallographic contrast is only about $5 \%$ of the total signal and is commonly observed between grains of different crystal orientation in a polycrystalline specimen, known as grain contrast or simply orientation contrast. In addition, the crystallographic contrast is visible inside grains containing low angle boundaries, kink bands, twins and defects substructure such as dislocation patterns [2].

In this study, a set of in-situ synchrotron x-ray diffraction deformation experiments were conducted at pressures in the range of 2 to $7 \mathrm{GPa}$ on forsterite olivine which is an important constituent of the upper mantle. Detailed experimental methodology is found in [3]. The ECCI was performed on specimens at 5 $\mathrm{keV}$ electron beam energy and $7 \mathrm{~mm}$ working distance. No coating was applied to the specimen surface prior to imaging. The microstructure after the in-situ synchrotron x-ray diffraction deformation experiment is shown in Fig. 1. The low magnification SE micrograph of sample assembly is shown in Fig. 1a. The forsterite olivine sample and top and bottom alumina pistons were indicated with arrows. The ECCI results from the forsterite olivine sample and alumina are shown in Fig. 1b and Fig. 1c, respectively. In Fig. 1b, the channeling contrast variations observed across the microstructure indicated the variations in local crystal orientation. In particular, a channeling contrast in the form of cross-shaped and intersecting contours appeared inside the grain indicated with a black square. The contours had a higher backscattering intensity compared to the rest of the grain and were terminated by high angle grain boundaries. 
Following a recent study of Kaboli et al. [4], the channeling contrast in the form of cross-shaped and intersecting contours observed in Fig. $1 \mathrm{~b}$ is identified as rotation contour contrast (RCC). This contrast was generated by the local rotation of the crystal about two rotation axes during deformation. The EBSD quantitative crystal orientation mapping was used to identify the crystallographic directions of the rotation axes. Deformation analysis using ECCI and EBSD techniques and quantitative results from the in-situ synchrotron $\mathrm{x}$-ray diffraction deformation experiments were used to understand the plasticity of forsterite olivine at each experimental conditions.

\section{References:}

[1] P C Burnley and D Zhang, J. Phys.: Condens. Matter. 20(2008), pp. 285201.

[2] D C Joy et al, Proceedings of the 5th annual scanning electron microscope symposium part I and part II, workshop on biological specimen preparation for scanning electron microscopy, (1972), pp. 97104.

[3] P C Burnley, American Minerologist. 100(2015), pp. 1424-1433.

[4] S Kaboli and R Gauvin, Ultramicroscopy. 154(2015), pp. 42-48.

[5] This research was sponsored in part by the National Nuclear Security Administration under the Stewardship Science Academic Alliances program through DOE Cooperative Agreement \#DENA0001982, and by the National Science Foundation under award NSF-EAR13613399. Synchrotron experiments were performed at the Advanced Photon Source which is supported by DOE-BES, under Contract No. DE-AC02-06CH11357 at Sector 6BM which is supported by the Consortium for Materials Properties Research in Earth Sciences under NSF cooperative agreement EAR 06-49658.

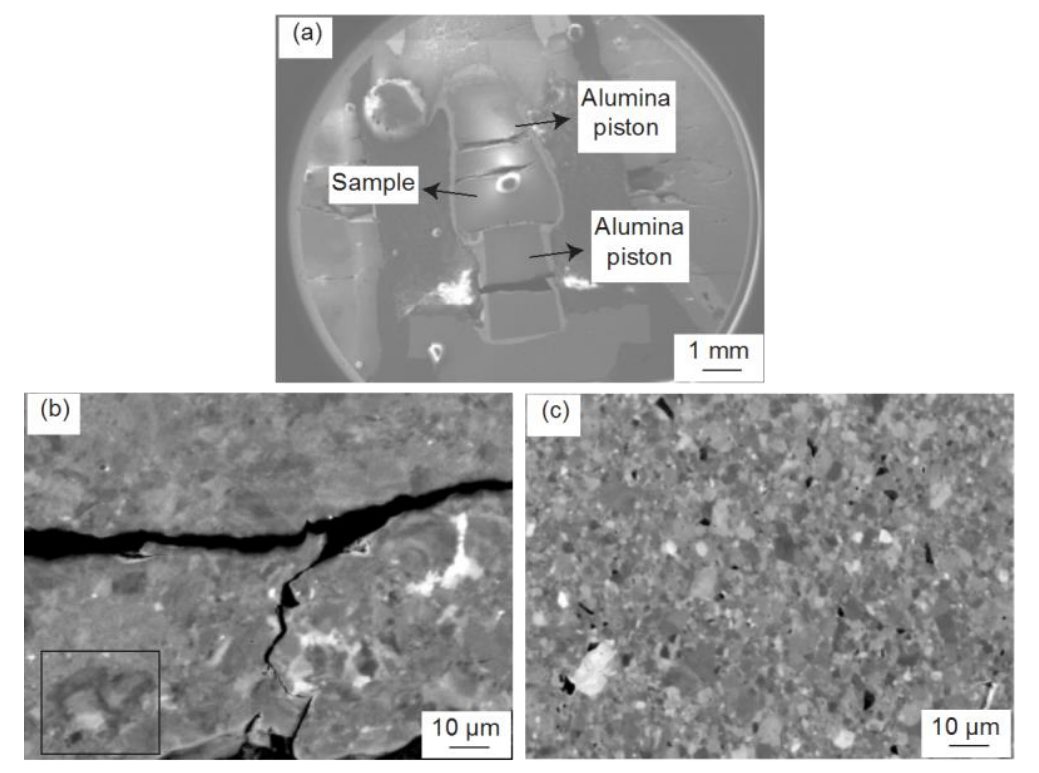

Figure 1. Microstructure of forsterite olivine after the in-situ synchrotron x-ray diffraction deformation experiment. (a) The secondary electron micrograph of the sample assembly with forsterite olivine sample and alumina pistons indicated with black arrows. (b) The backscattered electron micrograph of deformed forsterite olivine showing channeling contrast variations observed across the microstructure. An example of the rotation contour contrast in the form of cross-shaped and intersecting contours inside the grains was indicated with a black square. (c) The backscattered electron micrograph of deformed alumina. 